\title{
Inclusión de harina de Moringa oleifera en dietas para pollos de engorde
}

\author{
Rugel, D.O. ${ }^{1}$; Emén, M.F. ${ }^{2}$ \\ Departamentos de Producción Animal ${ }^{1}$ y Patología Animal ${ }^{2}$, Facultad Med. \\ Vet. y Zootecnia, Univ. Agraria del Ecuador. E-mail: drugel@uagraria.edu.ec
}

\begin{abstract}
Resumen
Rugel, D.O.; Emén, M.F.: Inclusión de harina de Moringa oleifera en dietas para pollos de engorde. Rev. Vet. 31: 1, 74-77, 2020. El propósito de la inclusión de la harina de M. oleifera en dietas para pollos de engorde fue determinar su efecto en relación a la ganancia de peso. Se utilizaron 15 pollos Broiler cobb 500 con un peso promedio inicial de 45 g. La metodología empleada fue un diseño experimental completamente al azar (DCA), distribuido en tres tratamientos con cinco repeticiones. Los tratamientos fueron $\mathrm{T}_{1}$ : balanceado comercial, $\mathrm{T}_{2}$ : balanceado con harina de moringa al $7 \%, \mathrm{y} \mathrm{T}_{3}$ : balanceado con harina de moringa al $15 \%$. Se registraron los pesos semanales de cada grupo, hasta los 42 días. Los resultados evidenciaron que la adición de $M$. oleifera al $7 \%$ constituye una materia prima viable para implementarla en dietas basadas en balanceados comerciales, siendo bien tolerado y logrando un incremento significativo en el peso final de las aves.
\end{abstract}

Palabras clave: pollos broiler, harina de moringa, alimentación, ganancia de peso.

\begin{abstract}
Rugel, D.O.; Emén, M.F.: Inclusion of Moringa oleifera flour in diets for broiler chickens. Rev. Vet. 31: 1, 74-77, 2020. The purpose of the inclusion of M. oleifera leaf meal in diets for broilers was to determine its effect in relation to weight gain. Fifteen chickens of Broiler cobb 500 breed with an initial average weight of $45 \mathrm{~g}$, were used. The methodology consisted in a completely randomized experimental design (DCR), distributed in three treatments with five repetitions. The treatments were $\mathrm{T}_{1}$ : balanced commercial diet, $\mathrm{T}_{2}$ : balanced diet with moringa flour at $7 \%$, and $\mathrm{T}_{3}$ : balanced diet with moringa flour at $15 \%$. The weekly weights of each group were recorded, up to 42 days. The results showed that the addition of $7 \% M$. oleifera flour is a viable supplement to implement in diets based on commercial balanced foods, well tolerated, achieving an increase in the final weight of the birds.
\end{abstract}

Key words: broiler chickens, moringa meal, feed, weight gain.

\section{INTRODUCCIÓN}

En la producción de pollos de engorde se ha utilizado como alimento tradicional al balanceado comercial, el cual ha sido elaborado por la industria alimentaria con estándares que poseen niveles definidos de nutrientes necesarios para la producción.

El costo del alimento representa el rubro más alto en el proceso productivo, por lo cual es un factor crítico para el sector industrial avícola ${ }^{8}$, motivo por el cual varias investigaciones se han enfocado en estudiar fuentes de alimentos alternativas que permitan reducir el costo de producción, manteniendo o mejorando las características de estas aves de consumo humano.

La nutrición de pollos de engorde Broiler Cobb 500 es de gran interés, dado que el crecimiento y el incremento del peso dependen de la alimentación que se le suministre $^{2}$. El balanceado comercial no proporciona los requerimientos nutricionales necesarios para la ali-

Recibido: enero 2020 / Aceptado: marzo 2020 mentación de los pollos, lo que ocasiona a largo plazo perjuicios en la economía del avicultor ${ }^{3}$.

La industria ecuatoriana produce alrededor de 200 millones de pollos en pie por año y aproximadamente entre 400 y 450 mil toneladas de carne de pollo, representando el consumo promedio de $32 \mathrm{~kg}$ por habitante ${ }^{11}$.

En la última década la demanda de pollos de engorde ha incrementado significativamente por el elevado contenido proteico que posee su carne ${ }^{11}$. Por ello se buscan nuevas formas de incorporar en la nutrición suplementos de bajos costos que ayuden a incrementar el peso ${ }^{7}$.

Moringa oleifera pertenece a la familia de árboles y arbustos Moringaceae, de distribución mundial en trópicos y subtrópicos; sin embargo, su origen se remonta a zonas del Agra y Oudh, al noroeste de la India y al sur de las montañas del Himalaya, Pakistán, Bangladesh y Afganistán ${ }^{4}$. En la última década se ha estudiado la influencia de M. oleifera como suplemento alimenticio en diferentes aves ${ }^{9}$. 
El cultivo de $M$. oleifera, además de proporcionar alta energía alimentaria, puede contribuir a prácticas agrícolas sostenibles, capaces de impulsar el desarrollo de las zonas rurales ${ }^{13}$. Sus hojas y vainas aportan vitaminas y minerales ${ }^{5}$, así como sus ramas, tallos y hojas proveen un alto contenido proteínas, azúcar y aceite en el caso de sus semillas ${ }^{4}$.

Debido al alto contenido de proteínas -alrededor del 27\%-, así como minerales y aminoácidos esenciales, se ha utilizado para complementar las dietas tradicionales de las aves de corral ${ }^{17}$. M. oleifera posee además altos niveles de fósforo, calcio, hierro y vitaminas $\mathrm{A}, \mathrm{B}$ y $\mathrm{C}^{1}$. El contenido de calcio y potasio, así como de vitaminas $\mathrm{A}$ y $\mathrm{C}$, se ha considerado que es mayor en relación a nutrientes como leche de vaca, zanahoria, naranja y plátano ${ }^{12}$. Al otorgar propiedades terapéuticas y nutritivas previene al pollo de afecciones en forma natural y lo beneficia en la reducción de fármacos para el tratamiento de las enfermedades que pueden persistir en el animal ${ }^{15}$.

La harina de M. oleifera posee importanes ventajas ya que los niveles de taninos y saponinas son mínimos y no posee inhibidores de tripsina ni lectina, por lo cual beneficia el crecimiento del pollo ${ }^{3}$. Si bien es digestible y palatable, si se emplea en cantidad mayor al $20 \%$ puede acelerar el metabolismo y provocar infartos ${ }^{11}$.

El objetivo de esta investigación fue determinar el nivel óptimo de inclusión de harina de $M$. oleifera en dietas comerciales, así como su efecto sobre la ganancia de peso en pollos broiler.

\section{MATERIAL Y MÉTODOS}

La investigación fue cuantitativa, con un diseño experimental completamente al azar (DCA), considerando tres tratamientos con cinco repeticiones cada uno. Las unidades experimentales fueron pollos Broiler de la línea Cobb 500, con peso referencial de $45 \mathrm{~g}$, machos procedentes de la misma incubadora, vacunados contra gripe aviar y sin presencia de enfermedades ni lesiones.

Cada grupo fue colocado en un galpón con luz incandescente, bebederos y comederos ad hoc para el suministro de agua y alimentos. En todos los galpones se colocó en el piso cascarilla de arroz ( $5 \mathrm{~cm}$ de espesor), para evitar la presencia de bacterias y parásitos, proceso que se repitió semanalmente.

Considerando el principio de aleatoriedad, se distribuyeron al azar cinco unidades experimentales en cada tratamiento. Todas las unidades pasaron por un período de adaptación de 7 días, y todas se manejaron con las mismas condiciones, siendo vacunadas contra New Castle y Gumboro.

A partir del octavo día se inició la alimentación de acuerdo a cada grupo. Al primer tratamiento ( $\mathrm{T}_{1}$ grupo control) se le suministró solo el balanceado comercial; al segundo tratamiento $\left(\mathrm{T}_{2}\right)$ se le suministró balanceado comercial con $7 \%$ de harina de moringa; y al tercer tratamiento $\left(\mathrm{T}_{3}\right)$ se le suministró balanceado comercial con $15 \%$ de harina de moringa.
Durante las 7 semanas del experimento se realizaron los pesajes de los pollos y se los registraron en gramos. A partir de la segunda semana del estudio se empezó a calcular la ganancia de peso de acuerdo a la siguiente fórmula:

$$
g p=p_{f}-p_{i}
$$

$g p$ : ganancia de peso en gramos

$p_{f}$ : peso del pollo de la semana actual

$p_{i}$ : peso del pollo de la semana anterior

El modelo estadístico aplicado fue:

$$
\begin{aligned}
& Y_{i j}=\mu+\gamma_{i}+\varepsilon_{i j} \\
& i=1,2,3 \quad j=1,2,3,4,5
\end{aligned}
$$

Donde:

$Y_{i j}$ : es la ganancia de peso en el $i-$ ésimo tratamiento y la $j$-ésima repetición

$\mu$ : es el efecto de la media general

$\gamma_{i}$ : es el efecto del $i$-ésimo tratamiento

$\varepsilon_{i j}$ : es el efecto del error experimental en el $i$-ésimo tratamiento y la $j$-ésima repetición

En el análisis estadístico se consideró la verificación del supuesto de normalidad a través de la prueba de Shapiro-Wilk y del supuesto de homogeneidad de varianzas a través de la prueba de Levene. El análisis comparativo se realizó mediante ANOVA de un factor considerando 5\% de nivel de significancia.

\section{RESULTADOS Y DISCUSIÓN}

En cinco de las seis mediciones, los animales que consumieron alimento balanceado con harina de moringa al 7\% obtuvieron mayor promedio de ganancia de peso; sin embargo, la menor dispersión se dio en el grupo que consumió balanceado con moringa al $15 \%$, como puede observarse en Tabla 1.

No obstante, el análisis de varianza determinó que solo en la última medición -al día 42- existieron diferencias significativas $(p<0,05)$, como se puede observar en la Figura 1. Durante toda la experiencia, la ganancia de peso del tratamiento que consumió balanceado con moringa al $15 \%$ fue menor que los otros tratamientos.

Tabla 1. Ganancias de peso (gramos)

\begin{tabular}{cccc}
\hline $\begin{array}{c}\text { semana } \\
\text { de } \\
\text { medición }\end{array}$ & $\begin{array}{c}\text { balanceado } \\
\text { M (DE) }\end{array}$ & $\begin{array}{c}\text { balanceado }+ \\
\text { Moringa al } \\
7 \% \text { M (DE) }\end{array}$ & $\begin{array}{c}\text { balanceado }+ \\
\text { Moringa al } \\
15 \% \text { M (DE) }\end{array}$ \\
\hline 2 & $193(84,88)$ & $206(54,28)$ & $157(19,73)$ \\
3 & $472(172,55)$ & $471(66,30)$ & $338(39,80)$ \\
4 & $582(196,22)$ & $652(74,19)$ & $523(34,85)$ \\
5 & $740(123,41)$ & $691(103,32)$ & $648(29,87)$ \\
6 & $715(61,80)$ & $755(77,82)$ & $616(50,52)$ \\
7 & $445(45,83)$ & $940(208,35)$ & $595(60,62)$ \\
\hline
\end{tabular}

M: media aritmética; DE: desviación estándar 


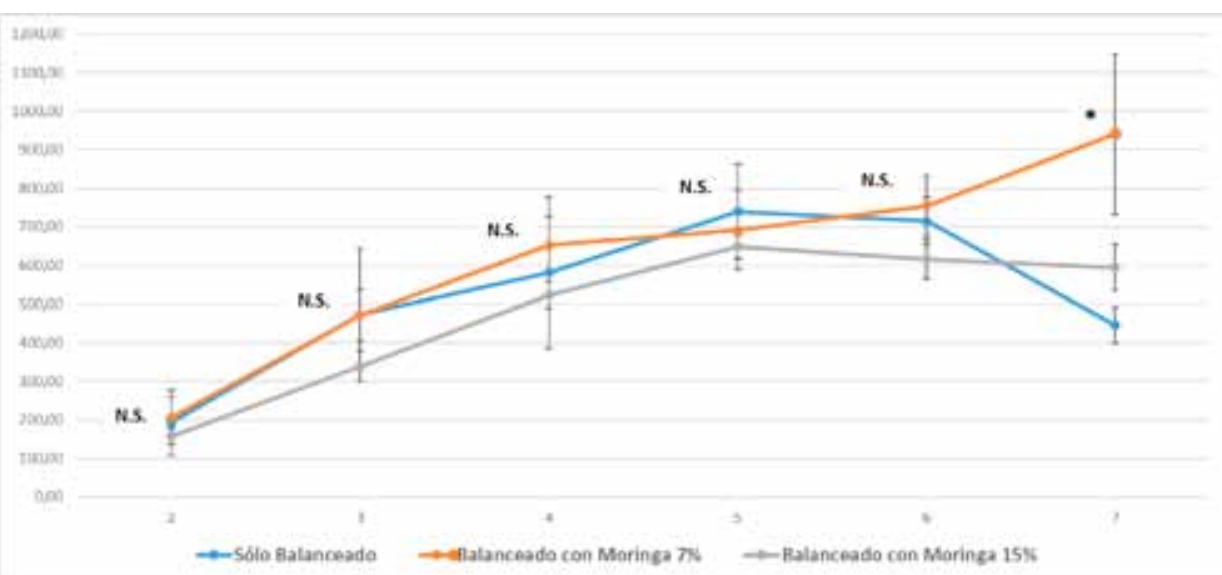

Figura 1. Precisión del experimento por cada tratamiento, en cada semana de medición. *Diferencias significativas con nivel de significancia del $5 \%$.

El resultado obtenido en esta investigacion resultó similar a los reportados por otros autores. La suplementación de harina de $M$. oleifera al $7 \%$ reveló diferencias significativas en la ganacia de peso, aunque concentraciones menores han sido reportadas aceptables, como en un estudio donde se determinó que concentraciones al $5 \%$ de $M$. oleifera fueron aceptables, sin afectar los índices hematológicos ${ }^{8}$. No obstante, otras investigaciones señalan que índices hasta del 7,5\% no incidieron de forma negativa en dichos parámetros ${ }^{10}$.

Un estudio llevado a cabo en Formosa, Argentina, señaló que la inclusión de harina de hojas de M. oleifera puede ser incorporadas de forma fiable hasta un $8 \%$ en la alimentación de pollos parrilleros. También se reportó una buena aceptacion en cuanto a palatabilidad del suplemento por parte de las aves, aunque ellas no presentaron diferencias significativas con el balanceado normal, tanto en el peso corporal como en el rendimiento productivo ${ }^{6}$.

Tal reducción del rendimiento puede ser debida a la presencia de fitatos, los cuales reducirían la biodisponibilidad de los minerales y disminuiría la digestibilidad de almidón y proteínas en los animales ${ }^{14}$.

Por otra parte, algunos investigadores han hallado que $M$. oleifera es un potencial recurso de alimentación para las aves de corral en Sudáfrica, ya que la ingesta entre 50 a $70 \mathrm{~g} / \mathrm{kg}$ reduciría los costos de alimentación e incluso mejoraría el rendimiento de la carcasa ${ }^{16}$.

A concentraciones de $7,5 \%$ se ha reportado la aparición de una coloración amarillenta en la carcasa 5 , debido a la presencia de betacarotenos en las hojas de $M$. oleifera, incluso cuando éstas hubieran sufrido proceso de deshidratación ${ }^{13}$. Este cambio en el color de la carcasa podría provocar el rechazo por parte del consumidor ${ }^{5}$.

Los resultados mostraron que la suplementación con harina de M. oleifera en concentraciones de hasta el $7 \%$ en dietas comerciales para pollos broiler, es beneficiosa y no afecta negativamente la ganancia de peso.

Agradecimientos. A los estudiantes de Medicina Veterinaria y Zootecnia: Lisette Alvarado, Danny Ca- brera, Jonathan Cruz, Boris Gómez y Harold Guamán, por su valioso aporte en el trabajo de campo realizado en esta investigación.

\section{REFERENCIAS}

1. Abou EZ, Sarmiento FL, Santos RR, Solorio SF. 2011. Efectos nutricionales de la inclusión dietética de harina de hojas de Leucaena leucocephala y Moringa oleifera en el comportamiento de gallinas. Rev Cub Cs Agric 45: 163170 .

2. Aviagen. 2009. Aviagen broiler breeders. http://es.aviagen. com/assets/Tech_Center/BB_Foreign_Language_Docs/ Spanish_TechDocs/smA-Acres-Guia-de-Manejo-delPollo-Engorde-2009.pdf

3. Cabezas DV. 2013. Programa Nacional Sanitario Avicola. Dirección de Sanidad Animal. Quito, Ecuador, Resolucion-071. www.agrocalidad.gob.ec.

4. Foidl N, Makkar H, Becker K. 2002. The potential of Moringa oleifera for agricultural and industrial uses. International Workshop, Dar-es-Salaam, Tanzania. http:// agritrop.cirad.fr/510176/

5. Gakuya DW, Mbugua PN, Kavoi B, Kiama SG. 2014. Effect of supplementation of Moringa oleifera leaf meal in broiler chicken feed. Int J Poultry Sci 13: 208-213.

6. Gómez NI, Rébak G, Fernández R, Sindik M, Sanz P. 2016. Comportamiento productivo de pollos parrilleros alimentados con Moringa oleifera en Formosa, Argentina. Rev Vet 27: 7-10.

7. Mufwaya UP, Kiatoko MH. 2016. Effet de substitution de tourteau palmiste par le foin de Moringa oléifera dans la ration, sur la croissance de poulet de chair de souche. Int $J$ Innov \& Applied Studies 15: 936-942.

8. Olugbemi T, Mutayoba S, Lekule F. 2010. Effect of moringa (Moringa oleifera) inclusion in cassava based diets fed to broiler chickens. Int J Poultry Sci 9: 363-367.

9. Olugbemi T, Mutayoba S, Lekule F. 2010. Evaluation of Moringa oleifera meaf meal inclusion in cassava chip based diet fed to laying birds. Liv Res for Rural Develop 22: 6, http://www.lrrd.org/lrrd22/6/olug22118.htm. 
10. Onu PN, Aniebo AO. 2011. Influence of Moringa oleifera leaf meal on the performance and blood chemistry of starter broilers. Intern J Food Agric \& Vet Sci 1: 38-44.

11. Orellana J. 2007. Corporacion Nacional de Avicultores del Ecuador-CONAVE. PDF: http://amevea-ecuador.org/ web_antigua/datos/AMEVEA, pag. 1-11.

12. Pérez A, Sánchez T, Armengol N, Reyes F. 2010. Características y potencialidad de Moringa oleifera, Lamark. Una alternativa para la alimentación animal. Pastos \& Forrajes 33: 1-16.

13. Raman JK, Alves CM, Gnansounou E. 2018. A review on moringa tree and vetiver grass. Potential biorefinery feedstocks. Biores Technol 249: 1044-1051.

14. Reddy NR, Sathe SK, Salunkhe DK. 1982. Phytates in legumes and cereals. Adv in Food Res 28: 1-92.
15. Sánchez KY, Cuadros AF, Peña MJ. 2016. Impacto que genera la utilización de Moringa oleifera en la producción de pollo. Rev Mundo FESC 12: 98-108.

16. Sebola NA, Mlambo V, Mokoboki HK, Muchenje V. 2015. Growth performance and carcass characteristics of three chicken strains in response to incremental levels of dietary Moringa oleifera leaf meal. Livestock Sci 178: 202208.

17. Zanu HK, Asiedu P, Tampuori M, Abada M, Asante I. 2012. Possibilities of using moringa (Moringa oleifera) leaf meal as a partial substitute for mishmeal in broiler chickens diets. J Anim \& Feed Res 2: 70-75. 